\title{
Adjusted Fireworks Algorithm Applied to Retinal Image Registration
}

\author{
Eva TUBA ${ }^{1}$, Milan TUBA ${ }^{1}$, Edin DOLICANIN ${ }^{2}$ \\ ${ }^{1}$ Graduate School of Computer Science, John Naisbitt University, Bul. umetnosti 29, Belgrade, 11070, Serbia. \\ etuba@acm.org; tuba@ieee.org \\ ${ }^{2}$ Dept. of Technical Sciences, State University of Novi Pazar, Vuka Karadzica bb, Novi Pazar, 36300, Serbia. \\ edin@np.ac.rs
}

\begin{abstract}
Image registration is an important problem in image processing, especially in medical imaging. It is a hard optimization problem with objective function that is not appropriate for deterministic mathematical optimization. In this paper we propose a bi-resolution algorithm for rigid image registration based on recent fireworks algorithm. We tested our approach on retinal images from standard benchmark datasets and compared the results with other approaches from literature. Our proposed algorithm succeeded in registering images in all cases, and was more accurate than other compared methods.
\end{abstract}

\section{Introduction}

Medical image processing is an important and widely researched scientific field [24], [20], [17] where image registration has significant applications. Image registration refers to the problem of finding a spatial relationship between two or more images, usually called reference image and floating images. This relationship can be described by linear transformations, also known as rigid transformations, or by non-linear (nonrigid, elastic) transformations.

Image registration techniques can be divided into two main groups: feature based and area based techniques [22]. Feature based techniques provide transformation which maps floating image to the reference image based on some common features between these two images. In [6] highly distinctive local feature descriptor named partial intensity invariant feature descriptor was proposed to describe a robust automatic retinal image registration. A method for automatic image registration through histogrambased image segmentation was proposed in [10], while geometrical features were used in [8]. In [9] curvelet transform was used to extract vessels and the extracted vessels from two modalities were registered together in two stages. In [7] a method based on the block matching algorithms was proposed. General adaptive neighbourhoods were used for block similarity. The main issue that limits feature based techniques is finding reliable feature correspondence between images [14].

Area based techniques search for optimal parameters for transformation of the floating image in order to align it with the reference image. These techniques, unlike feature based ones, operate on pixel values.
Advantage of area based techniques is that they do not need any user interaction in identifying landmarks, nor segmentation algorithms. On the other hand, they are slower but that can be improved by identifying regions of interest. Pixels outside these regions can be ignored. In [15] a general method that uses bootstrap resampling in order to estimate the uncertainty of area based image registration algorithms was presented. In [4] automatic image-to-image registration method was introduced where a topologically regular grid of tie points was imposed within the overlapping region of the images.

Intensity based similarity of two images for area based techniques can be defined in numerous ways. Some of the most commonly used similarity measurements are the sum of squared intensity differences, cross correlation, mutual information, etc. [29]. In [32] a method for the rigid registration of two $\mathrm{CT}$ brain bones images was proposed where mutual information and genetic algorithm were used. The main task in the area based techniques is to optimize the similarity metrics. This problem represents a hard optimization problem. In the past two decades stochastic optimization algorithms, especially swarm intelligence, were successfully used for such problems.

One of the earliest swarm intelligence algorithms was particle swarm optimization (PSO), which has been applied to the image registration problem. In [12] approach based on modified particle swarm optimization and wavelet decomposition was proposed for rigid image registration. In [19] an enhanced binary particle swarm optimization technique was used to 
optimize the control points collected by directional finding algorithm to retrieve the binary registered result. In [31], particle swarm optimization algorithm was adjusted for optimization of transformation parameters with hybrid mutual information as a similarity measurement. Revised quantum-behaved particle swarm optimization and diversity-controlled quantum PSO were applied to medical image registration in [41]. In [36] a PSO algorithm was combined with sequential quadratic programming for rigid image registration. In [30] the artificial bee colony, more recent stochastic optimization algorithm, was used for rigid image registration. Another relatively new swarm intelligence algorithm, firefly algorithm, was used for searching maximal similarity measurement in [37] and [35]. Paper [5] deals with the time complexity issue by using multithreading parallel processing technique.

In this paper we propose to use one of the latest swarm intelligence algorithms, the fireworks algorithm (FWA), for finding optimal parameters of rigid transformation for retinal image registration of $2 \mathrm{D}$ images.

The resolution of retinal images from the used dataset is $565 \times 584$ pixels. This resolution is too large for finding the optimal parameters for wide search range so we proposed one resolution reduction in order to narrow the search range. In this paper the optimal reduction factor for retinal images as well as optimal parameters for the fireworks algorithm were empirically determined.

The rest of the paper is organized as follows. A mathematical model for image registration is presented in Section 2. In Section 3 bi-resolution image technique is explained. Section 4 provides details about fireworks algorithm. Our proposed algorithm and para-meter settings are presented in Section 5. Experimental results and comparative analysis are given in Section 6. At the end, in Section 7 conclusions and future work are presented.

\section{Mathematical Model for Image Registration}

Algorithms for image registration start with two images, one labelled as reference and one as floating image. Floating image should be transformed to align with the reference image.

Next, it is necessary to choose criterion function that determines the similarity between two images.
The last step is to optimize the transformation so that the stopping criteria are satisfied. Stopping criteria can be the number of iterations for optimization algorithm or desired similarity between images.

Image registration refers to the mapping that is the best match between two or more images of the same scene taken at different times or taken by different sensors. Assuming that $B$ is a floating image that should to be aligned with the reference image $A$, mathematically mapping can be defined as:

$$
A(x, y)=\zeta\left(B\left(T_{\alpha}(x, y)\right)\right.
$$

where $\zeta$ is the intensity calibration function and $T_{\alpha}$ is transformation function that maps spatial coordinates $x$ and $y$ to new spatial coordinates $x$ ' and $y$ ' with the transformation parameters $\alpha$ :

$$
\left(x^{\prime}, y^{\prime}\right)=T_{\alpha}(x, y) \text {. }
$$

Image registration determines spatial transformation which maximizes similarity of the reference and floating images. Previous description of image registration can be represented by the following equation:

$T^{*}=\arg \max O\left(A, T_{\alpha}(B)\right)$,

where $T_{\alpha}$ is one possible transformation and $O$ represents the objective function which is actually the similarity measurement. In this case, represents transformation with parameters $\alpha$ which maximizes the value of the objective function over all possible transformations. For different purposes different transformations are used. One type of transformations $T$ are linear transformations that include translation, rotation, scaling and other affine transformations. Common name for these transformations is rigid transformations. On the other hand, there are non-linear transformations, also called elastic or non-rigid transformations, which include radial basis functions, physical continuum models and large deformation models. Elastic transformations map curve lines to other curve lines. In this paper we used rigid transformations that include translation and rotation. These transformations are defined by two translation parameters $t_{x}$ and $t_{y}$ and angle of rotation $\theta$. Transformation formula can be written in the matrix form:

$$
T=\left|\begin{array}{ccc}
\cos (\theta) & -\sin (\theta) & t_{x} \\
\sin (\theta) & \cos (\theta) & t_{y} \\
0 & 0 & 1
\end{array}\right|
$$


Finding optimal transformation depends on the chosen similarity measure. Similarity measure represents indicator of closeness between two images of feature values in the case of feature based techniques, or intensity values in the case of area based techniques. One of the simplest similarity measures is the mean of squared differences between intensity values. Another often used similarity measure is cross-correlation or normalized cross-correlation [29]. One of the most used similarity measures is mutual information that is defined by the following expression [13]:

$$
\mathrm{I}(\mathrm{A}, \mathrm{B})=\mathrm{H}(\mathrm{A})+\mathrm{H}(\mathrm{B})-\mathrm{H}(\mathrm{A}, \mathrm{B}) \text {, }
$$

where $A$ and $B$ represent reference and floating images, respectively, $H(A)$ and $H(B)$ are image entropies and $H(A, B)$ is the joint entropy. Entropy of an image can be calculated using the following equation:

$$
\mathrm{H}(\mathrm{A})=-\frac{1}{\mathrm{~N}} * \sum_{\mathrm{i}=1}^{\mathrm{N}} \mathrm{p}_{\mathrm{i}} \log p_{i},
$$

where $N$ is the number of pixels in the image and the $p_{i}$ is the normalized number of pixels with intensity level $i$. In [34] entropy correlation coefficient instead of mutual information was proposed. Also, normalized mutual information is a common choice for similarity measure. It is defined as:

$$
\mathrm{I}(\mathrm{A}, \mathrm{B})=\frac{\mathrm{H}(\mathrm{A})+\mathrm{H}(\mathrm{B})}{\mathrm{H}(\mathrm{A}, \mathrm{B})} \text {. }
$$

\section{Bi-resolution Image Registration}

The evaluation of the similarity measurement can be computationally very expensive. In order to reduce the computational time, one often used approach is to perform registration first on images with the reduced resolution. The resulting transform is then used as a starting point for the next registration round which is performed on images of higher resolution [18].

Including the multiresolution technique in image registration has two advantages. It increases performance and it also improves the optimization stability because it smooths out the local minimums.

The simplest version of multiresolution is bilevel resolution, where only one reduction of the resolution is done. In this paper we used images with resolution that was not very high, so there was no need for more than one reduction of the resolution. The resolution reduction can be done by simple averaging. Regions of the size $n$ $\mathrm{x} n$ become one pixel whose intensity level is set to the average value of the corresponding $n * n$ pixels. Appropriate region size was determined empirically.

In this paper we used image with reduced resolution to find the initial range for search space. The first step of the algorithm is to search for transformation parameters in wide range of possible values using the image with reduced resolution. Results of this search are used to reduce the search space for the full resolution image where fine search is done around values from the previous step.

\section{Fireworks Algorithm}

One of the latest swarm intelligence algorithms, proposed in 2010 by Tan and Zhu, is the fireworks algorithm (FWA) [25]. As a result of the wide use of the fireworks algorithm for different optimization problem, many different modifications, enhancements and hybridizations of the algorithm were proposed. Authors of the original fireworks algorithm continued to work on its improvement. The results are four upgraded versions of the FWA, enhanced FWA [39], corporative framework for FWA [40], FWA with enhanced interaction and the latest update is guided FWA (GFWA) [16]. In [38] they proposed another improvement of the enhanced fireworks algorithm (EFWA) based on an adaptive dynamic local search mechanism. Fireworks algorithm was used in many different applications, for example, in [28] it was used for RFID network planning, in [3] for constrained portfolio optimization problem, in $[2,26]$ for wireless sensor networks localization problem, in [27] for support vector machine parameters tuning, etc.

Fireworks algorithm simulates explosion of a firework that produces a shower of sparks around the place where firework is set off and this phenomenon was used as an inspiration for this algorithm. For exploitation and exploration phenomena of badly and well manufactured fireworks are used. Badly manufactured fireworks are characterized by small number of sparks scattered in space, which is used to provide exploration. On the other hand, well manufactured fireworks have a lot of sparks centralized around the place where firework was set off which corresponds to exploitation. In fireworks algorithm the number of the sparks for firework $X_{i}$ is calculated using the following equation [16]: 


$$
\lambda_{i}=m \frac{y_{\max }-f\left(X_{i}\right)+\eta}{\sum_{j=1}^{n}\left(y_{\max }-f\left(X_{j}\right)\right)+\eta},
$$

where parameter $m$ controls the overall number of sparks generated by $n$ fireworks, $y_{\max }=\max \left(f\left(X_{i}\right)\right),(i=1,2, \ldots, n)$ represents the worst firework in the population, $f$ is the objective function while $\eta$ represents a small constant used to avoid division by zero.

It is necessary to determine the amplitude of the explosion. Explosion amplitude in iteration $i$ is modelled for each firework by the following equation [16]:

$$
A_{i}=\hat{A} \frac{f\left(X_{i}\right)-y_{\min }+\eta}{\sum_{j=1}^{n}\left(f\left(X_{j}\right)-y_{\min }\right)+\eta},
$$

where $\hat{A}$ is a constant parameter which controls the explosion amplitude, $y_{\min }=\min \left(f\left(X_{i}\right)\right)$, $(i=1,2, \ldots, n)$ represents the best firework in the population and $\eta$ is a small constant used to avoid division by zero. Using the calculated amplitude, change for new position for component $k$ will be:

$$
\Delta X_{k}=A_{i} \times \operatorname{rand}(-1,1) \text {. }
$$

When comparison with other algorithms is done it should be noted that FWA does not count the number of iterations but objective function evaluations since the number of sparks in each iteration can be very different.

\section{The Proposed Fireworks Algorithm Adjusted for Image Registration Problem}

In this paper we considered rigid transformations, translation and rotation, according to Eq. (4). For similarity measure we used normalized mutual information given by Eq. (7). Objective function for image registration that uses mutual information for similarity measurement has numerous local maxima hence global optimization search is needed [34], [35], [12]. Deterministic methods are powerless in such situations and swarm intelligence algorithms are state-of-the-art tools that are known to quickly produce at least good suboptimal solutions [33]. We adopted and adjusted recent swarm intelligence fireworks algorithm [16].

Normalized mutual information for two images cannot exceed the value that is obtained when two images are equal. However, when the picture is translated by just one pixel or rotated by only $1^{\circ}$, normalized mutual information changes significantly. For example, for one test image the maximal normalized mutual information was 1.000 for equal images and it dropped to 0.681 when the picture was translated along $x$ axis by 1 pixel. Further changes are minor compared to this one and getting smaller as translation increases. For example normalized mutual information for translation along $x$ axis by 10 pixels was 0.607 and for translation by 20 pixels was 0.590. Similar situation is for rotation, normalized mutual information from the maximum of 1.000 dropped to 0.648 for rotation of $1^{\circ}$.

Such objective function is very difficult since the global optimum is hidden in a very narrow well, the rest of the search space being relatively flat with numerous local optima. The search algorithm has very little opportunity to use exploitation unless it has at least one hit in the narrow area around global optimum.

Based on these observations, the fireworks algorithm was adjusted. At the beginning, wide search is needed in order to cover search space with aim to find that narrow space where global maximum is. Number of candidate solutions (sparks) need to be much larger than for most applications in order to speedup the initial hit close to the global optimum. However, amplitude coefficient should be small since outside the narrow area of the global optimum it does not help to do exploitation, closer or wider. Once that narrow space has been found, exploitation should be very fine with small steps. In order to provide fine steps in later iterations, Eq. (10) was adjusted. Instead of changing amplitude constantly by random number from the range $[-1$, 1], we decrease the range depending on the evaluation (iteration) number. In the beginning, search range should be wider, but after small number of evaluations it should become much smaller. In our proposed method this was achieved by reducing the range quadratically with the progress of evaluations. We empirically established that after $20 \%$ of evaluations the range should decrease to 0.5 and after $90 \%$ of evaluations it should decrease to 0.1 . This resulted in the replacement of Eq. (10) by:

$$
\begin{aligned}
& \Delta X_{k}=A_{i} \times \operatorname{rand}(-s(t), s(t)) \\
& s(t)=2.14\left(\frac{t}{t \text { Max }}\right)^{2}-2.93\left(\frac{t}{t \text { Max }}\right)+1.00
\end{aligned}
$$

where $t$ is the number of current evaluation and tMax is the maximum number of evaluations. 
Search range for transformation parameters must be wide enough to cover all possible situations. In this paper, we assumed that the search interval for $t_{x}$ and $t_{y}$ is $[-100,100]$, while for $\Theta$ is $[-50$, 50]. For this search range, the proposed fireworks algorithm was not able to find the optimal solution when image in full resolution was used. In Table 1. results for registration of images shown in Fig. 1 are presented. The original image was translated for $t_{x}=60, t_{y}=70$ and rotated by $\Theta=30^{\circ}$, the origin is at the top left corner.

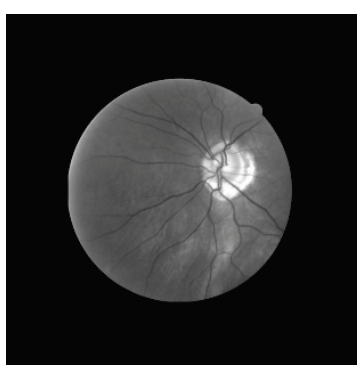

(a) Reference image

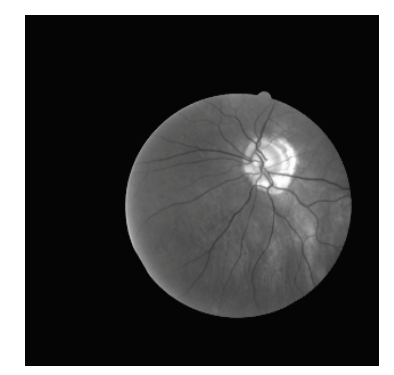

(b) Floating image
Figure 1. Test images (a) original, (b) transformed by $t_{x}=60, t_{y}=70$ and $\theta=30^{\circ}$

For each experiment the algorithm was run 30 times with different random seeds. The mean, standard deviation as well as minimal and maximal results are listed in the table. Average execution times are also shown.

From Table 1 it can be seen that increasing the number of evaluations does not help, found solutions are far from the real values of the transformation parameters. Computational times are significant since the objective function processes the whole image and it requires about 500 times more time than standard benchmark functions.

Table 1. Estimated values for $t_{x}, t_{y}$, and $\Theta$ for different number of iteration on full resolution for actual $\mathrm{t}_{\mathrm{x}}=60, \mathrm{t}_{\mathrm{y}}=70, \Theta=30^{\circ}$ and $\mathrm{t}_{\mathrm{x}}, \mathrm{t}_{\mathrm{y}} \in[-100,100]$, $\Theta \in[-50,50]$

\begin{tabular}{|c|c|c|c|c|}
\hline Evals & & $\mathrm{t}_{\mathrm{x}}$ & \multicolumn{1}{c|}{$\mathrm{t}_{\mathrm{y}}$} & \multicolumn{1}{c|}{$\Theta$} \\
\hline \multirow{3}{*}{1000} & Avg. & 61.849 & 49.389 & 21.649 \\
\cline { 2 - 5 } & Std. & 25.127 & 52.668 & 15.984 \\
\cline { 2 - 5 } $159 \mathrm{~s}$ & Min & 4.639 & -55.278 & -12.004 \\
\cline { 2 - 5 } & Max & 87.281 & 95.279 & 34.915 \\
\hline \multirow{3}{*}{2000} & Avg. & 49.487 & 68.447 & 29.576 \\
\cline { 2 - 5 } & Std. & 18.847 & 4.522 & 1.573 \\
\cline { 2 - 5 } $312 \mathrm{~s}$ & Min & 25.870 & 60.601 & 27.557 \\
\cline { 2 - 5 } & Max & 69.764 & 72.286 & 31.230 \\
\hline \multirow{3}{*}{3000} & Avg. & 51.055 & 59.352 & 19.899 \\
\cline { 2 - 5 } & Std. & 24.449 & 19.953 & 19.836 \\
\cline { 2 - 5 } $473 \mathrm{~s}$ & Min & 7.716 & 24.133 & -15.361 \\
\cline { 2 - 5 } & Max & 67.577 & 70.081 & 30.002 \\
\hline
\end{tabular}

On the other hand, if the search interval is narrow, the proposed adjusted FWA finds good solutions in small number of evaluations as it can be seen in Table 2. If the search space is narrowed to \pm 2 , in only 500 evaluations transformation parameters were found with acceptable accuracy. Execution time was 76s. Accuracy between 750 iterations and 1000 iteration is almost the same. In order to narrow the search space for parameters, one resolution reduction is used.

Table 2. Estimated values for $t_{x}, t_{y}$, and $\Theta$ for different number of iteration on full resolution for actual $\mathrm{t}_{\mathrm{x}}=60, \mathrm{t}_{\mathrm{y}}=70, \Theta=30^{\circ}$ and $\mathrm{t}_{\mathrm{x}}, \mathrm{t}_{\mathrm{y}} \in\left[\mathrm{t}_{\mathrm{x}, \mathrm{y}} \mathrm{-}\right.$,

$$
\left.t_{x, y}+2\right], \Theta \in[\Theta-2, \Theta+2]
$$

\begin{tabular}{|c|c|r|r|r|}
\hline Evals. & & \multicolumn{1}{|c|}{$\mathrm{t}_{\mathrm{x}}$} & \multicolumn{1}{c|}{$\mathrm{t}_{\mathrm{y}}$} & \multicolumn{1}{|c|}{$\Theta$} \\
\hline \multirow{3}{*}{500} & Avg. & 60.497 & 70.261 & 29.610 \\
\cline { 2 - 5 } & Std. & 0.542 & 0.309 & 0.878 \\
\cline { 2 - 5 } $76 \mathrm{~s}$ & Min & 59.876 & 69.907 & 28.040 \\
\cline { 2 - 5 } & Max & 61.113 & 70.719 & 30.018 \\
\hline \multirow{3}{*}{750} & Avg. & 60.031 & 69.976 & 29.998 \\
\cline { 2 - 5 } $121 \mathrm{~s}$ & Std. & 0.029 & 0.063 & 0.003 \\
\cline { 2 - 5 } & Min & 60.005 & 69.870 & 29.993 \\
\cline { 2 - 5 } & Max & 60.075 & 70.024 & 30.000 \\
\hline \multirow{3}{*}{1000} & Avg. & 59.992 & 70.020 & 30.004 \\
\cline { 2 - 5 } & Std. & 0.043 & 0.023 & 0.003 \\
\cline { 2 - 5 } $159 \mathrm{~s}$ & Min & 59.924 & 69.995 & 30.000 \\
\cline { 2 - 5 } & Max & 60.028 & 70.046 & 30.009 \\
\hline
\end{tabular}

The purpose of resolution reduction is to find an approximate solution and to use it to narrow the search space for the full resolution image. When the resolution is reduced, the search is faster, but also less precise because a number of original pixels merge into one. This operation has the effect of smoothing filter and edges are attenuated. With reduced edges it is possible to use a simpler fitness function like the sum of the absolute differences:

$$
f(A, B)=\sum_{i=1}^{n} \sum_{j=1}^{m}|A(i, j)-B(i, j)|
$$

where $\mathrm{A}$ and $\mathrm{B}$ are reference and floating image of the size $n \times m$. Such similarity metrics is unusable when many edges are present since misalignment for only one pixel produces huge differences at edges. Possibility to use this simpler objective function with reduced resolution images is important since it allows for significant computation time reduction.

The reason is that when normalized mutual information objective function defined by Eq. (7) is used, execution time is not significantly 
reduced when the image resolution is reduced. Computation of that objective function includes only one pass through the image for building histogram.

That operation depends on the resolution but it involves only one simple increment operation.

After that the histogram is used, involving more complex operations and the size is fixed to 256 for single image and to $256 \times 256$ for joint entropy, regardless of the resolution. That makes significant reduction of computational time by reducing resolution impossible.

Another adjustment for images with reduced resolution has been made in the proposed algorithm. Since parameters obtained by reduced images are unprecise, fine exploitation is not necessary and on the other hand more exploration is needed because of the wide search space. Based on this, instead of using Eq. (11) from the beginning of the algorithm, in the first 1000 evaluations regular FWA was used.

In Table 3. experimental results for different resolution reductions are shown. In the case of $k \mathrm{x} k$ resolution reduction, transformation parameters were also reduced by $k$ and later rescaled back for comparison. The computational time is significantly reduced by using reduced resolution and simpler objective function: 12 times for $4 \mathrm{x} 4$ reduction.

Table 3. Estimated values for $t_{x}, t_{y}$, and $\Theta$ for different resolution for actual $t_{x}=60, t_{y}=70, \Theta=30^{\circ}$, and $t_{x}, t_{y} \in[-100,100], \Theta \in[-50,50]$

\begin{tabular}{|c|c|c|c|c|}
\hline Reduction & & $t_{x}$ & $t_{y}$ & $\theta$ \\
\hline \multirow{3}{*}{$2 \times 2$} & Avg. & $\overline{559.852}$ & 66.139 & 29.750 \\
\hline & Std. & 0.547 & 8.639 & 0.353 \\
\hline & Min & 59.309 & 50.703 & 29.413 \\
\hline $81 \mathrm{~s}$ & Max & 60.732 & 70.308 & 30.117 \\
\hline \multirow{4}{*}{$4 \times 4$} & Avg. & 60.167 & 69.785 & 29.772 \\
\hline & Std. & 0.491 & 0.247 & 0.113 \\
\hline & Min & 59.725 & 69.423 & 29.607 \\
\hline & Max & 60.870 & 69.969 & 29.845 \\
\hline \multirow{4}{*}{$6 \times 6$} & Avg. & 59.445 & 73.322 & 30.623 \\
\hline & Std. & 0.263 & 3.003 & 1.561 \\
\hline & Min & 59.220 & 71.009 & 29.656 \\
\hline & Max & 59.876 & 77.460 & 33.381 \\
\hline \multirow{4}{*}{$8 \times 8$} & Avg. & 58.337 & 71.122 & 30.857 \\
\hline & Std. & 1.107 & 2.637 & 1.647 \\
\hline & Min & 57.433 & 68.173 & 29.810 \\
\hline & Max & 59.770 & 72.653 & 32.090 \\
\hline \multirow{4}{*}{$10 \times 10$} & Avg. & 58.780 & 68.452 & 29.060 \\
\hline & Std. & 0.278 & 3.715 & 1.381 \\
\hline & Min & 58.407 & 61.868 & 28.407 \\
\hline & Max & 59.067 & 70.871 & 30.329 \\
\hline
\end{tabular}

Moreover, with the image in reduced resolution optimal parameters were found with acceptable accuracy. In Table 1 it has been shown that in full resolution the algorithm was not able to find solution. From Table 3 it can be seen that reduction $2 \times 2$ is also not enough, but reduction $4 \times 4$ gives good results. Further reduction of the resolution makes results less precise and standard deviation larger because too many pixels were merged into one.

Based on the results from Table 2 and Table 3 it can be concluded that the best combination is to use $4 \times 4$ reduction in the first step with 3000 evaluations. This will find intermediate solution such that error and $3 \sigma$ combined are less than 2 .

In the second step search range for transformation parameters can be reduced to \pm 2 of parameters obtained in the first step.

Parameters for the fireworks algorithm were set empirically. As usually, the number of fireworks was set to 5. Explosion amplitude was set to 40 and minimal and maximal number of sparks were set to 2 and 40, respectively.

Optimization parameters were translation parameters $t_{x}$ and $t_{y}$ and angle of rotation $\theta$, all three as floating point numbers. This floating point model covers real world imaging where misalignment is continuous and also makes our results comparable to [34].

We included few preprocessing steps. Images are of the rectangular shape and when rotation is performed some of the information from the image would be lost. Since we need to have all the information, at the beginning we extended the image by adding a black frame large enough to save all original pixels after rotation. Image background was set to be black using the appropriate threshold, so the frame was the same color as the background. Empirically determined threshold was adequate in this case but more elaborate techniques are available [11], [1]. For images without borders (Fig. 3 and Fig. 4) we used the inner part of the image that is common to both reference and floating image.

For rotation, each square pixel can in new position partially cover up to six pixels in the original grid (four for real translation) so we attributed original pixel intensity value to all affected pixels, with appropriate weights. Such transformation is not exactly reversible. 


\section{Experimental Results}

The proposed algorithm was implemented using Matlab R2016a. All experiments were performed on the platform with the following characteristics: Intel ${ }^{\circledR}$ Core $^{\mathrm{TM}}$ i7-3770K CPU at $4 \mathrm{GHz}, 8 \mathrm{~GB}$ RAM, Windows 10 Professional OS.

Parameters for fireworks algorithm were determined empirically, using theoretical guidance as explained in the previous sections.

Our proposed algorithm was tested on DRIVE dataset described in [23] publicly available at www.isi.uu.nl/Research/Databases/DRIVE/. This database contains 40 images of retina. Original images are in the RGB color model. In [14] only green channel was used because it had the highest contrast between vessels and the background. It is also possible to transform the image to grey scale. We tested both, green channel images and grey images and also concluded that the green channel image is slightly better since vessels are more pronounced. In order to test our algorithm we transformed each picture by known transformation parameters and compared parameters estimated by the fireworks algorithm to the original ones.

In [34] bacterial multiple colony chemotaxis (BMCC) was proposed as the optimization algorithm and tri-linear interpolation and partial volume distribution for elimination of local extrema. For retinal image transformed by the same parameters results were reported for three different similarity measures, entropy correlation coefficient (ECC), average of ECC obtained from tri-linear interpolation and partial volume distribution and their novel combination of these ECCs. Parameters of transformation were $t_{x}=5, t_{y}=10$ and rotation for $\theta=20^{\circ}$ (Fig. 2). The best registration was achieved with their proposed combination. They reported $t_{x}=4.920, t_{y}=9.988$ and $\theta=20.002^{\circ}$. With our proposed algorithm we successfully obtained better results. For translation parameters we obtained $t_{x}=5.069$, $t_{y}=9.984, \theta=20.000^{\circ}$.

In another example from [34] parameters used for making the floating image were $t_{x}=3, t_{y}=3$ and $\theta=15^{\circ}$ (Fig. 3). Best approximation was obtained using their proposed method and they reported the following transformation parameters: $t_{x}=2.883, \quad t_{y}=3.010 \quad$ and $\theta=15.002^{\circ}$.

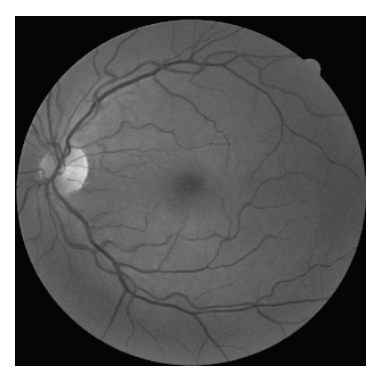

(a) Reference image

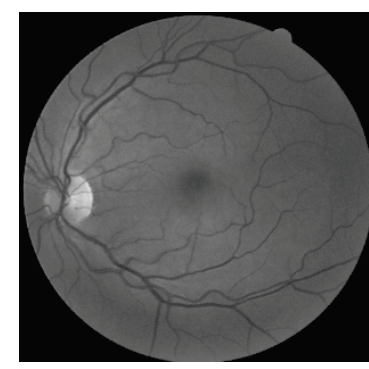

(b) Floating image
Figure 2. Image pair 1, transformation parameters $t_{x}=5, t_{y}=10$ and $\theta=20^{\circ}$

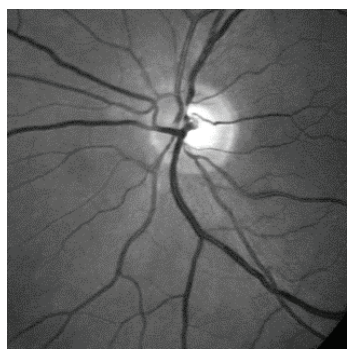

(a) Reference image

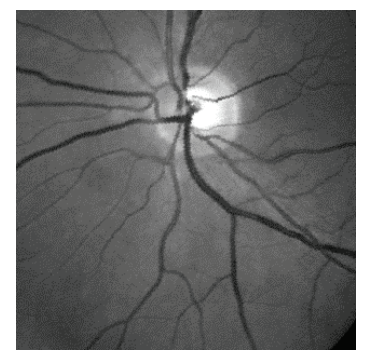

(b) Floating image
Figure 3. Image pair 2, transformation parameters $t_{x}=3, t_{y}=3$ and $\theta=15^{\circ}$

The results from [34] (BMCC) were also compared with three other optimization algorithms from the literature (chaotic particle swarm optimization (CPS), elite preservation genetic algorithm (EGA) and ant colony algorithm (ACA)) and they found that their algorithm was better. Our proposed algorithm found the best registration for the parameters $t_{x}=2.986, t_{y}=2.999$ and $\theta=15.008^{\circ}$. It can be noticed that our proposed method found slightly better results than the best results from [34] and indirectly better than other compared algorithms which are presented in Table 4. Additionally, results in [34] were obtained using multi-resolution scheme, while our results were obtained with only one resolution reduction.

For the last example transformation parameters were set to $t_{x}=-5, t_{y}=5$ and $\theta=9^{\circ}$ (Fig. 4). In [34] the following results were reported: $t_{x}=-5.1744$, $t_{y}=4.7834$ and $\theta=9.0011^{\circ}$, while our proposed algorithm found optimal parameters for values $t_{x}=-5.0020, t_{y}=4.9967$ and $\theta=8.9826^{\circ}$ which is again better.

Table 4. Comparison between our proposed algorithm and approaches from [34]

\begin{tabular}{|c|c|c|c|}
\hline & $\mathbf{t}_{\mathbf{x}}$ & $\mathbf{t}_{\mathbf{y}}$ & $\boldsymbol{\Theta}$ \\
\hline Exact results & 3 & 3 & 15 \\
\hline EGA & 2.893 & 3.092 & 15.180 \\
\hline ACA & 2.891 & 3.113 & 15.176 \\
\hline CPSO & 2.819 & 3.016 & 15.226 \\
\hline BMCC & 2.883 & 3.010 & $\mathbf{1 5 . 0 0 2}$ \\
\hline FWA & $\mathbf{2 . 9 8 6}$ & $\mathbf{2 . 9 9 9}$ & 15.008 \\
\hline & & & \\
\hline
\end{tabular}




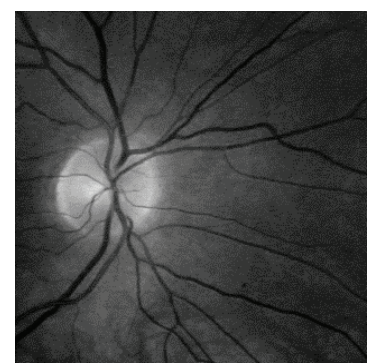

(a) Reference image

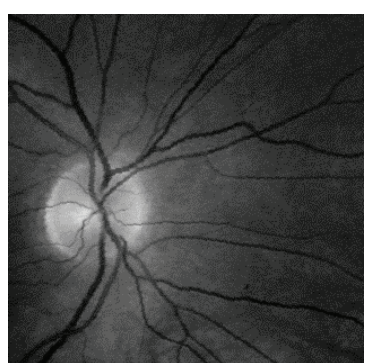

(b) Floating image
Figure 4. Image pair 3, transformation parameters

$$
t_{x}=-5, t_{y}=5 \text { and } \theta=9^{\circ}
$$

In Table 5. we summarized results for our proposed algorithm and results from [34] for this three examples.

Table 5. Comparison of results obtained by our proposed algorithm and results from [34]

\begin{tabular}{|c|c|c|c|c|c|}
\hline $\begin{array}{c}\text { Img } \\
\text { pair }\end{array}$ & & $\begin{array}{r}\text { REA } \\
\mathbf{L}\end{array}$ & BMCC & $\begin{array}{c}\text { FWA } \\
\text { step 1 }\end{array}$ & $\begin{array}{c}\text { FWA } \\
\text { step 2 }\end{array}$ \\
\hline 1 & $\mathrm{t}_{\mathrm{x}}$ & 5 & 4.920 & 4.860 & $\mathbf{5 . 0 6 9}$ \\
& $\mathrm{t}_{\mathrm{y}}$ & 10 & $\mathbf{9 . 9 8 8}$ & 9.681 & 9.984 \\
& $\Theta$ & 20 & 20.002 & 20.027 & $\mathbf{2 0 . 0 0 0}$ \\
\hline 2 & $\mathrm{t}_{\mathrm{x}}$ & 3 & 2.883 & 4.001 & $\mathbf{2 . 9 8 6}$ \\
& $\Theta$ & 15 & $\mathbf{1 5 . 0 0 2}$ & 15.567 & 15.008 \\
\hline 3 & $\mathrm{t}_{\mathrm{x}}$ & -5 & -5.175 & -4.279 & $\mathbf{- 5 . 0 0 2}$ \\
& $\mathrm{t}_{\mathrm{y}}$ & 5 & 4.783 & 5.188 & $\mathbf{4 . 9 9 7}$ \\
& $\Theta$ & 9 & $\mathbf{9 . 0 0 1}$ & 10.472 & 8.983 \\
\hline
\end{tabular}

In [21] an algorithm for retinal image registration using expectation maximization with mutual information was proposed. Integers were used for translation parameters so these parameters were reported without error. For rotation of $20^{\circ}$ largest error was 1.425 while the average error was 0.204. With our proposed algorithm error For angle in all cases was less than 0.134 which means that our proposed algorithm also outperformed method proposed in [21]. Run times for our algorithm were 40s for step 1 (from Table 3) and 159s for step 2 (from Table 2) while papers [34] and [21] did not report run times.

Larger values for parameters may not make physical sense considering characteristics of image producing equipment and limitations to patients position and movement, but we tested for parameters $t_{x}=50, t_{y}=80$ and $\theta=60^{\circ}$ in order to prove the quality of our proposed algorithm. For this example, our proposed algorithm successfully found $t_{x}=50.0036, t_{y}=79.9661$ and $\theta=60.0001^{\circ}$ with the same run time.

We tested our algorithm with same images and different transformation parameters and also with different images from the mentioned dataset and achieved perfect registration in all cases which leads to the conclusion that the proposed fireworks algorithm is completely appropriate for retinal image registrations.

\section{Conclusion}

For the hard optimization problem of rigid image registration we adjusted recent fireworks algorithm and tested it on retinal images from datasets used in literature. Since the algorithm could not find solutions using full resolution images, appropriate resolution reduction was determined that facilitates initial coarse results and parameters were tuned so that the two stages of the procedure cooperate optimally. Normalized mutual information was substituted by simpler sum of the absolute differences as objective function for reduced resolution. We compared our approach with 6 other techniques from literature and it outperformed all of them. Our algorithm succeeded in registering images in all cases, including large displacement. That establishes fireworks algorithm as a superior tool for rigid retinal image registration. We did not use the hierarchical approach with multiple resolutions so in the future that can further improve results, mostly the execution time.

\section{Acknowledgement}

This research was supported by the Ministry of Education, Science and Technological Development of the Republic of Serbia, Grant No. III-44006.

\section{REFERENCES}

1. Alihodzic, A. \& Tuba, M. (2014). Improved Bat Algorithm Applied to Multilevel Image Thresholding. The Scientific World Journal, 2014, Article ID 176718, pp. 1-16.

2. Arsic, A., Tuba, M. \& Jordanski, M. (2016). Fireworks Algorithm Applied to Wireless Sensor Networks Localization Problem. In 2016 IEEE Congress on Evolutionary Computation (CEC), pp. 4038-4044. 
3. Bacanin, N. \& Tuba, M. (2015). Fireworks Algorithm Applied to Constrained Portfolio Optimization Problem. In 2015 IEEE Congress on Evolutionary Computation (CEC.), pp. 1242-1249.

4. Bunting, P., Labrosse, F. \& Lucas, R. (2010). A Multi-Resolution Area-Based Technique for Automatic Multi-Modal Image Registration. Image and Vision Computing, 28(8), pp. 1203-1219.

5. Chakraborty, S., Ghosh, S., Chatterjee, S., Chowdhuri, S., Ray, R. \& Dey, N. (2014). Rigid Image Registration Using Parallel Processing. In IEEE International Conference on Circuits, Communication, Control and Computing (I4C), pp. 237-241.

6. Chen, J., Tian, J., Lee, N., Zheng, J., Smith, R. T. \& Laine, A. F. (2010). A Partial Intensity Invariant Feature Descriptor for Multimodal Retinal Image Registration. IEEE transactions on bio-medical engineering, 57(7), pp. 1707-1718.

7. Debayle, J. \& Presles, B. (2016). Rigid Image Registration by General Adaptive Neighborhood Matching. Pattern Recognition, 55, pp. 45-57.

8. Gharabaghi, S., Daneshvar, S. \& Sedaaghi, M. H. (2013). Retinal Image Registration Using Geometrical Features. Journal of digital imaging, 26(2), pp. 248-258.

9. Golabbakhsh, M. \& Rabbani, H. (2013). Vessel-Based Registration of Fundus and Optical Coherence Tomography Projection Images of Retina Using a Quadratic Registration Model. IET Image Processing, 7(8), pp. 768-776.

10. Goncalves, H., Goncalves, J. A. \& CorteReal, L. (2011). HAIRIS: A Method for Automatic Image Registration Through Histogram-Based Image Segmentation. IEEE transactions on image processing, 20(3), pp. 776-789.

11. Hassanien, A. E., Abdelfattah, M., Amin, K. M. \& Mohamed, S. (2015). A Novel Hybrid Binarization Technique for Images of Historical Arabic Manuscripts, Studies in Informatics and Control, 24(3), pp. 271-282.

12. Hu, W., Chen, A. L. \& Song, L. L. (2011). An Approach for Rigid Image Registration Based on Wavelet Decomposition and Modified Particle Swarm Optimization. International Journal of Modelling,
Identification and Control, 14(4), pp. 272-278.

13. Jaeyong, J., Murray, L. \& Bonhwa, K. (2016). Hybrid Retinal Image Registration Using Mutual Information and Salient Features. IEICE Transactions on Information and Systems, 99(6), pp. 1729-1732.

14. Khan, M. K. \& Nystrom, I. (2010). A Modified Particle Swarm Optimization Applied in Image Registration. In 20th IEEE International Conference on Pattern Recognition, pp. 2302-2305.

15. Kybic, J. (2010). Bootstrap Resampling for Image Registration Uncertainty Estimation Without Ground Truth. IEEE Transactions on Image Processing, 19(1), pp. 64-73.

16. Li, J., Zheng, S. \& Tan, Y. (2017). The Effect of Information Utilization: Introducing a Novel Guiding Spark in The Fireworks Algorithm. IEEE Transactions on Evolutionary Computation, 21(1). pp. 153-166.

17. Luca, R. (2016). Clustering-based Human Locomotion Parameters for Motion Type Classification. Studies in Informatics and Control, 25(3), pp. 353-362.

18. Nejati, M. \& Pourghassem, H. (2014). Multiresolution Image Registration in Digital X-Ray Angiography with Intensity Variation Modeling. Journal of medical systems, 38(2), pp. 1-10.

19. Palraj, P. \& Vennila, I. (2016). Retinal Fundus Image Registration via Blood Vessel Extraction Using Binary Particle Swarm Optimization. Journal of Medical Imaging and Health Informatics, 6(2), pp.328-337.

20. Popescu, D., Ilie, C., Laptoiu, D., Hadar, A. \& Barbur, R. (2016). Web Based Collaborative Platform for Personalized Orthopaedic Applications, Studies in Informatics and Control, 25(4), pp.517-526.

21. Reel, P. S., Dooley, L. S., Wong, K. P., \& Börner, A. (2013). Robust Retinal Image Registration Using Expectation Maximisation with Mutual Information. In 2013 IEEE International Conference on Acoustics, Speech and Signal Processing, pp. 1118-1122.

22. Saxena, S. \& Singh, R. K. (2014). A Survey of Recent and Classical Image Registration Methods. International Journal of Signal Processing, Image Processing and Pattern Recognition, 7(4), pp.167-176. 
23. Staal, J., Abràmoff, M. D., Niemeijer, M., Viergever, M. A. \& Ginneken, B. (2004). Ridge-based Vessel Segmentation in Color Images of the Retina. IEEE Transactions on Medical Imaging, 23(4), pp. 501-509.

24. Stojak, A., Tuba, E. \& Tuba M. (2016) Framework for Abnormality Detection in Magnetic Resonance Brain Images, In IEEE $24^{\text {th }}$ Telecommunications Forum TELFOR, pp. 687-690.

25. Tan, Y. \& Zhu, Y. (2010). Fireworks Algorithm for Optimization. In International Conference in Swarm Intelligence, LNCS, 6145, pp. 355-364. Springer.

26. Tuba, E., Tuba, M. \& Beko, M. Node Localization in Ad Hoc Wireless Sensor Networks Using Fireworks Algorithm. In 5th International Conference on Multimedia Computing and Systems (ICMCS'16), pp. 1-7.

27. Tuba, E., Tuba, M. \& Beko, M. (2016). Support Vector Machine Parameters Optimization by Enhanced Fireworks Algorithm. Advance in Swarm Intelligence, LNCS, 9712. pp. 526-534. Springer.

28. Tuba, M., Bacanin, N. \& Beko, M. (2015). Fireworks Algorithm for RFID Network Planning Problem. In 25th International Conference Radioelektronika, pp. 440-444. IEEE.

29. Valsecchi, A., Damas, S. \& Santamaria, J. (2013). Evolutionary Intensity-Based Medical Image Registration: A Review. Current Medical Imaging Reviews, 9(4), pp. 283-297.

30. Wang, S. (2011). Artificial Bee Colony Used for Rigid Image Registration. International Journal of Research and Review in Soft and Intelligent Computing, 1(2), pp. 35-38.

31. Wei, B., Zhao, Z. \& Peng, X. (2011). Spatial Information Based Medical Image Registration Using Mutual Information. Journal of Multimedia, 6(3), pp. 236-243.

32. Wei, W., Liu, L., Hu, Z. Q. \& Zhou, Y. J. (2014). Rigid Medical Image Registration Based on Genetic Algorithms and Mutual Information. Applied Mechanics and Materials, 665, pp. 712-717.
33. Yang, X. S., Cui, Z., Xiao, R., Gandomi, A. H., \& Karamanoglu, M. (Eds.). (2013). Swarm Intelligence and Bio-Inspired Computation: Theory and Applications. Newnes, Elsevier Insights, pages 421 .

34. Yudong, Z. \& Lenan, W. (2008). Multiresolution Rigid Image Registration Using Bacterial Multiple Colony Chemotaxis. In 5th International Conference on Visual Information Engineering, pp. 528-532.

35. Zhang, Y. \& Wu, L. (2012). A Novel Method For Rigid Image Registration Based on Firefly Algorithm. International Journal of Research and Reviews in Soft and Intelligent Computing (IJRRSIC), 2(2), pp. 141-146.

36. Zhang, Y. \& Wu, L. (2012). Rigid Image Registration by PSOSQP Algorithm. Advances in Digital Multimedia, 1(1), pp. 4-8.

37. Zhang, Y. \& Wu, L. (2012). Rigid Image Registration Based on Normalized Cross Correlation and Chaotic Firefly Algorithm. International Journal of Digital Content Technology and its Applications, 6(22), pp. 129-140.

38. Zheng, S., Janecek, A., Li, J. \& Tan, Y. (2014). Dynamic search in fireworks algorithm. In 2014 IEEE Congress on Evolutionary Computation (CEC), pp. 3222-3229.

39. Zheng, S., Janecek, A. \& Tan, Y. (2013). Enhanced fireworks algorithm. In 2013 IEEE Congress on Evolutionary Computation, pp. 2069-2077. IEEE.

40. Zheng, S., Li, J., Janecek, A. \& Tan, Y. (2015). A Cooperative Framework for Fireworks Algorithm. IEEE/ACM Transactions on Computational Biology and Bioinformatics, PP(99). pp.1-13.

41. Zhou, D., Sun, J., Lai, C. H., Xu, W., \& Lee, X. (2011). An Improved Quantum-Behaved Particle Swarm Optimization and Its Application to Medical Image Registration. International Journal of Computer Mathematics, 88(6), pp. 1208-1223. 